\title{
Ascending Pharyngeal Artery Collateral Circulation Simulating Internal Carotid Artery Hypoplasia
}

\author{
R. G. Quisling ${ }^{1}$ and J.F. Seeger ${ }^{2}$ \\ Departments of Radiology, ${ }^{1}$ Neuroradiology Section of the University of Florida, and ${ }^{2}$ University of Michigan, USA
}

\begin{abstract}
Summary. Complete occlusion of the cervical segment of the internal carotid artery may result in a collateral circuit between an enlarged ascending pharyngeal artery and the intracranial segment of the internal carotid artery. This anastomosis may simulate a severely stenotic or hypoplastic internal carotid artery. Differentiation between these entities is particularly important if carotid endarterectomy for relief of stenosis is contemplated.
\end{abstract}

Key words: Ascending pharyngeal artery - Hypoplastic carotid artery - Collateral circulation

When an ascending pharyngeal artery serves as a collateral between the external and internal carotid circulations, it may simulate a hypoplastic or collapsed extracranial internal carotid artery. This is illustrated in two case presentations, and the therapeutic implications are discussed.

\section{Case 1}

A 60-year-old man presented with left-sided amaurosis fugax and transient ischemic attacks referable to his left cerebral hemisphere. Cerebral angiography shows complete occlusion of the extracranial segment of the internal carotid artery and prominent external-to-internal carotid artery collateral channels, including a markedly enlarged ascending pharyngeal artery (Figs. 1, 2 and 3).

\section{Case 2}

An 18-year-old woman was treated for a middle cerebral artery aneurysm at age 8 . She has had

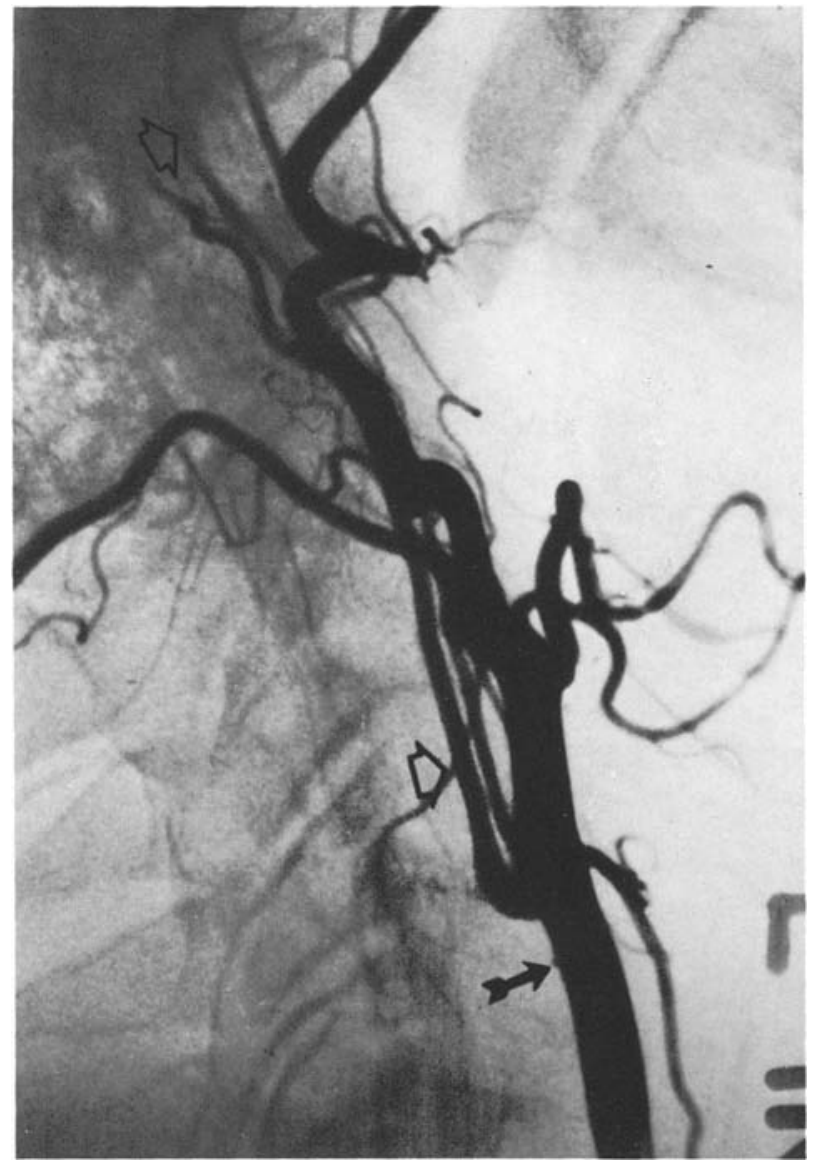

Fig. 1. (Case 1) Lateral projection of a left common carotid angiogram demonstrates an enlarged ascending pharyngeal-tointernal carotid artery anastomosis (open arrows) simulating a hypoplastic or collapsed internal carotid artery. A small dimple on the common carotid artery probably represents the residual lumen of the totally occluded true internal carotid artery (closed arrow) 


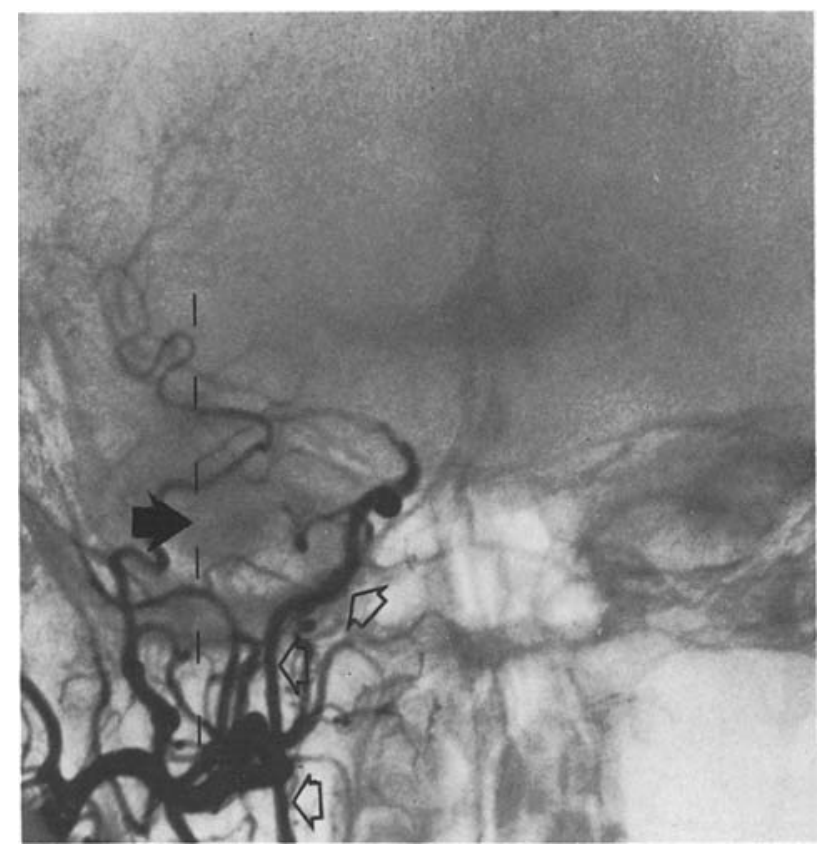

Fig. 2. (Case 1) AP left common carotid angiogram is projected to the right, following side-to-side reversal, to facilitate comparison with Figure 1. The hypertrophied ascending pharyngeal artery (open arrows) is located medial to the vestibular line. This artery probably penetrates the base of the skull through the foramen lacerum and anastomoses with branches of the artery of the inferior cavernous sinus. The genu of the intrapetrous carotid should be juxtaposed to the vestible (solid arrow)

increasing headaches but is otherwise asymptomatic. Cerebral angiography was performed in order to exclude an additional aneurysm. There is complete occlusion of the extracranial segment of the internal carotid artery and a filling defect in the distal supraclinoid portion of the internal carotid artery, most likely representing an intraluminal clot. As in Case 1, there is a prominent anastomosis between the ascending pharyngeal artery and the cavernous segment of the internal carotid artery (Figs. 4 and 5).

\section{Anatomy}

The normal course of the internal carotid artery is well known [3, 9]. Variations in the course of the petrosal segment of the internal carotid artery usually involve lateral displacement of the genu, even into the hypotympanum, while medial displacement has not been reported as an anatomic variant $[2,5,7]$. The course of the major trunk of the ascending pharyngeal artery closely parallels that of the internal carotid artery except that the former is more medially placed as it approaches the skull base when viewed in an AP projection $[1,6]$. Although it does give off an

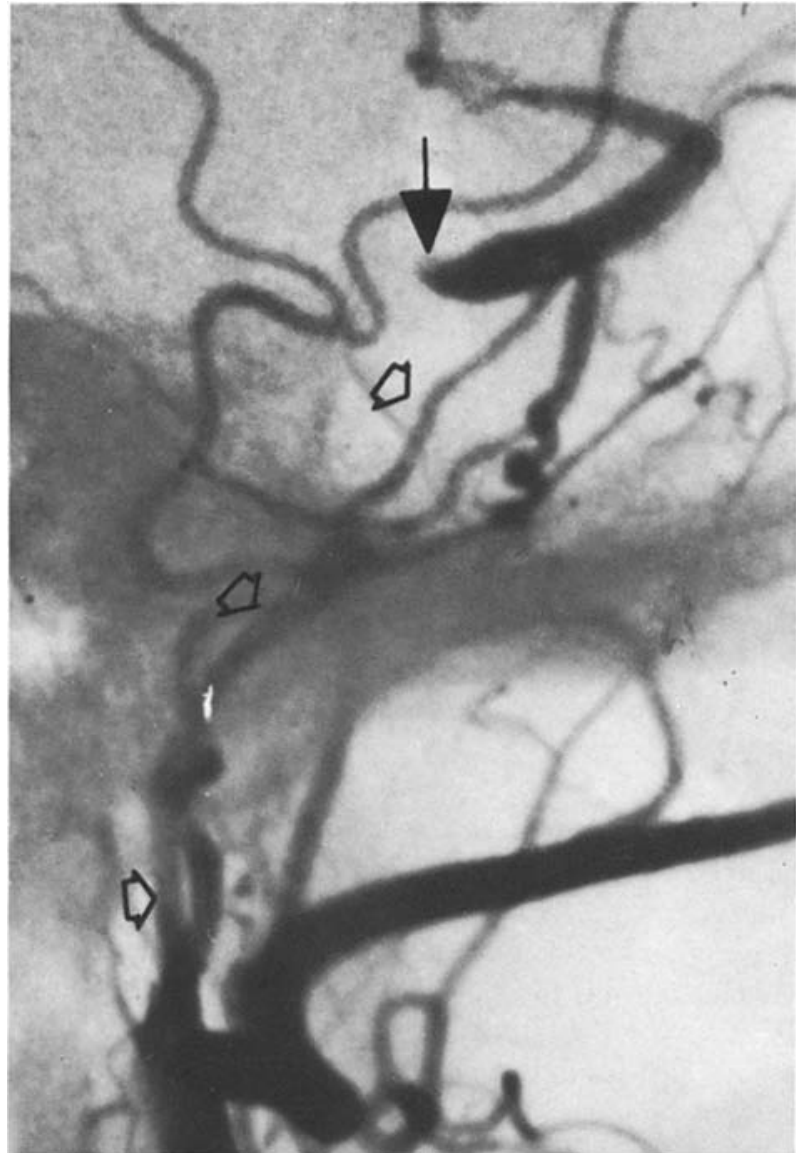

Fig. 3. (Case 1) Lateral filming of the head during the left common carotid angiogram shows the anastomosis of the ascending pharyngeal artery with branches of the cavernous internal carotid artery (open arrows). The stump of the remaining patent internal carotid artery is evident (solid arrow)

inferior tympanic branch, the parent artery terminates in the preclival nasopharynx. Distal branches of the ascending pharyngeal artery anastomose in the nasopharynx with branches of the internal carotid artery including the vidian artery, and arteries which pass through the foramen lacerum and foramen ovale. These latter two arteries are usually derived from the posterior division of the artery to the inferior cavernous sinus [4]. The potential collateral between the ascending pharyngeal artery and the artery to the inferior cavernous sinus is the subject of this paper.

\section{Discussion}

The lumen size of the internal carotid artery is related to multiple factors, including intraluminal arterial pressure. A significantly smaller-than-normal 


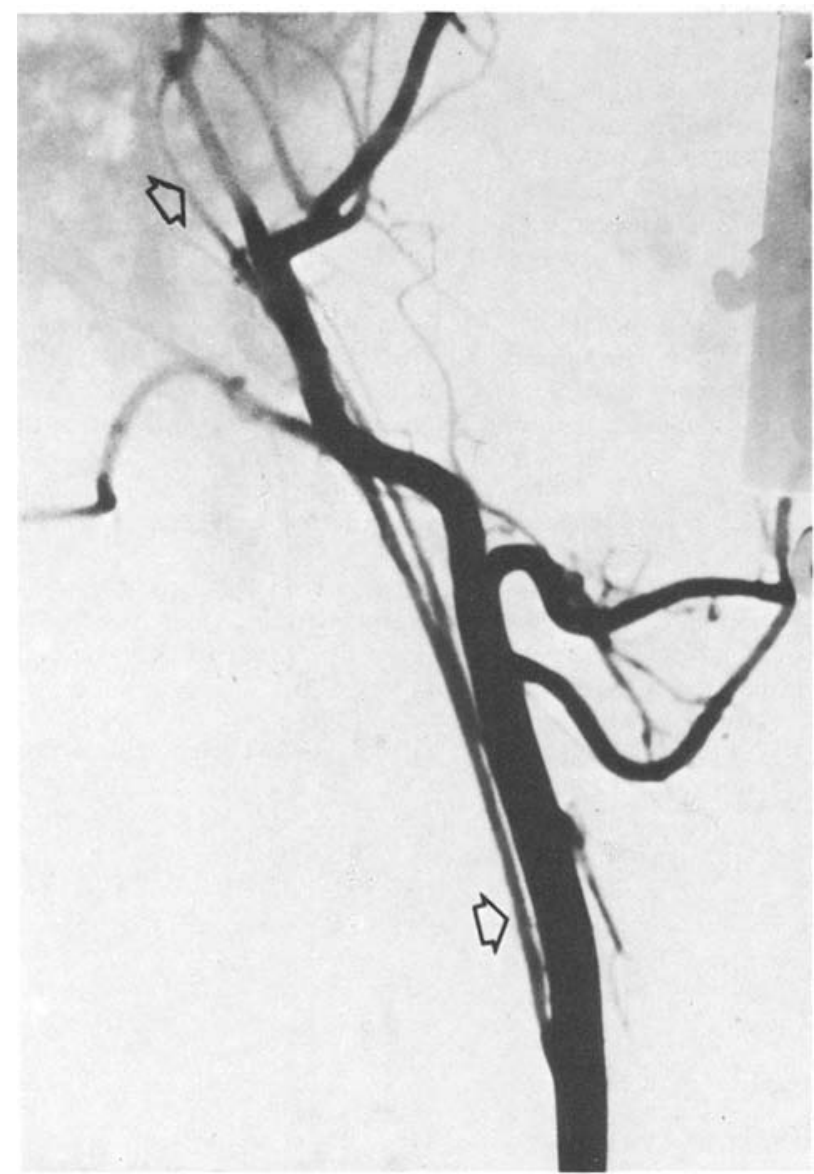

Fig. 4. (Case 2) Lateral projection of a common carotid angiogram demonstrates an apparent collapsed or hypoplastic internal carotid artery (open arrows), which in reality is an enlarged ascending pharyngeal artery

internal carotid artery may result from developmental hypoplasia. Whe have also seen several cases of high grade atheromatous stenosis of the origin of the internal carotid artery resulting in a collapsed arterial lumen.

It is well known that potential extracranial to intracranial collateral pathways may enlarge as a result of extracranial, internal carotid artery occlusion [8]. Under these circumstances the ascending pharyngeal-to-cavernous internal carotid artery collateral may increase to a size sufficient to be confused with either a hypoplastic, or even a stenotic and collapsed internal carotid artery. Differentiation is of particular importance if surgical attempts to increase cerebral blood flow are considered.

The ability to distinguish between a much smaller-than-normal internal carotid artery and a greatly enlarged ascending pharyngeal artery with collateral flow necessitates precise knowledge of their respective anatomic locations. Differentiation generally is

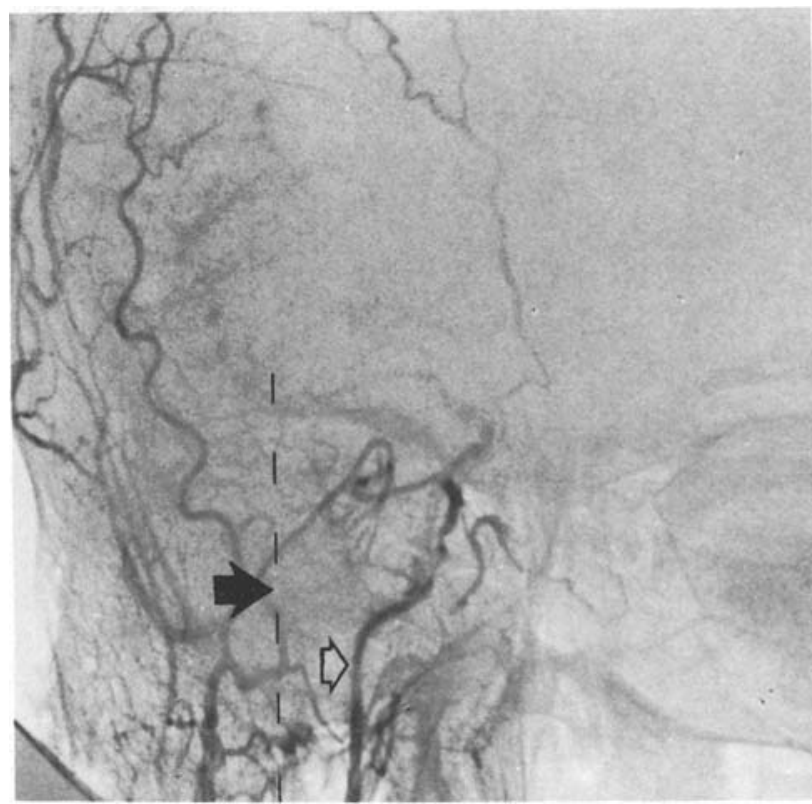

Fig. 5. (Case 2) The AP projection demonstrates the medial placement of this artery relative to the expected intrapetrous course of an internal carotid artery. Labeled structures include the vestibule (solid arrow), vertical mid-vesibular line (dashed line), ascending pharyngeal artery (open arrow)

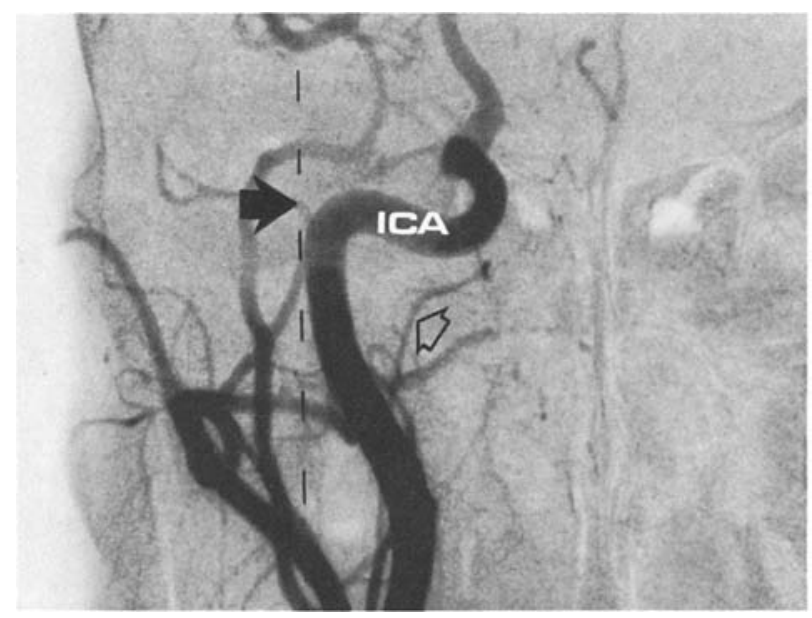

Fig. 6. AP projection of a right common carotid angiogram demonstrates the normal relationship between the course of the intrapetrous internal carotid artery (ICA) and the ascending pharyngeal artery (open arrow). The lateral margin of the vertical segment of the internal carotid artery lies adjacent to the hypotympanic portion of the middle ear, defined by a vertical line drawn through the vestibule (solid arrow). The ascending pharyngeal artery is normally situated at least one centimeter medial to the vertical vestibular line 
difficult on lateral projection. However, on AP projection, there are clear-cut anatomic differences. The intrapetrous portion of the internal carotid artery has three distinct segments including a vertical segment, a genu, and a horizontal segment. Also, the lateral margin of the genu approaches the hypotympanic portion of the middle ear, which may be approximated by extending a vertical line down from the vestibule [5] (Fig. 6).

The ascending pharyngeal collateral does not have three distinctly comparable segments and most important, its lateral margin does not reach the hypotympanic area (Figs. 2 and 5).

The advent of superficial temporal to middle cerebral artery microsurgical anastomoses now offers a feasible means of improving cerebral circulation. Selection of an appropriate surgical approach is based upon accurate angiographic assessment of the status of the internal and external carotid arteries. If the internal carotid artery lumen is merely collapsed distal to a high grade atheromatous stenosis at its origin, then removal of the atheromatous plaque ordinarily would seem to provide the best opportunity to improve the cerebral circulation, namely, via the internal carotid artery. However, in the absence of localized stenosis, endarterectomy of an enlarged ascending pharyngeal artery providing collateral flow to the intracranial carotid circulation would be contraindicated. In such a case, a superficial temporal to middle cerebral artery bypass procedure could still be considered in the symptomatic patient.

\section{References}

1. Allen, W. E., III, Kier, E. L., Rothman, S. L. G.: The maxillary artery in craniofacial pathology. Am. J. Radiol. 121, 124-138 (1974)

2. Goldman, N. C., Singleton, G. T., Holly, E. H.: Aberrant carotid artery. Arch. Otolaryngol. 94, 269-273 (1971)

3. Gray, H.: Anatomy of the human body. Philadelphia: Lea and Febiger 1976

4. Harris, F.S., Rhoton, A.L., Jr.: Anatomy of the cavernous sinus: a microsurgical study. J. Neurosurg. 45, 169-180 (1976)

5. Lapayowker, M. S., Liebman, E. P., Ronis, M. L., Safer, J. N.: Presentation of the internal carotid artery as a tumor of the middle ear. Radiology 98, 293-297 (1971)

6. Lasjaunias, P., Moret, J.: The ascending pharyngeal artery: normal and pathological radio-anatomy. Neuroradiology 11, 77-82 (1976)

7. Lie, T. A.: Congenital anamolies of the carotid arteries, pp. 95-105. Amsterdam: Excerpta Medica Foundation 1967

8. Margolis, M. T., Newton, T. H.: Collateral pathways between cavernous portion of the internal carotid and external carotid arteries. Radiology 93, 834-836 (1969)

9. Potter, G. D., Graham, M. D.: The carotid canal. Radiol. Clin. North Am. 12, 483-489 (1970)

Received: July 1979

Dr. R. G. Quisling

University of Florida Medical Center

Department of Radiology

Gainesville, FL 32601 USA 